
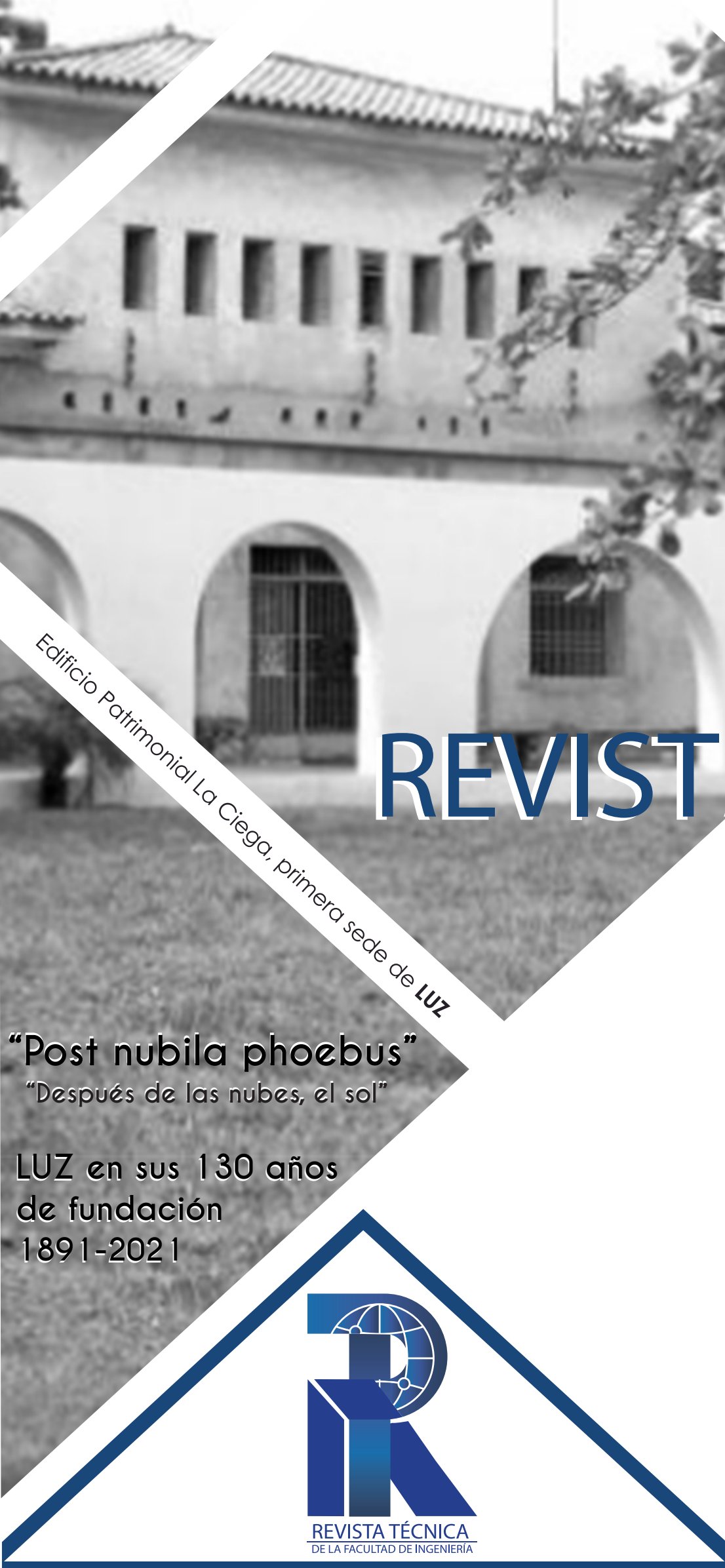

, $18,4,2$,
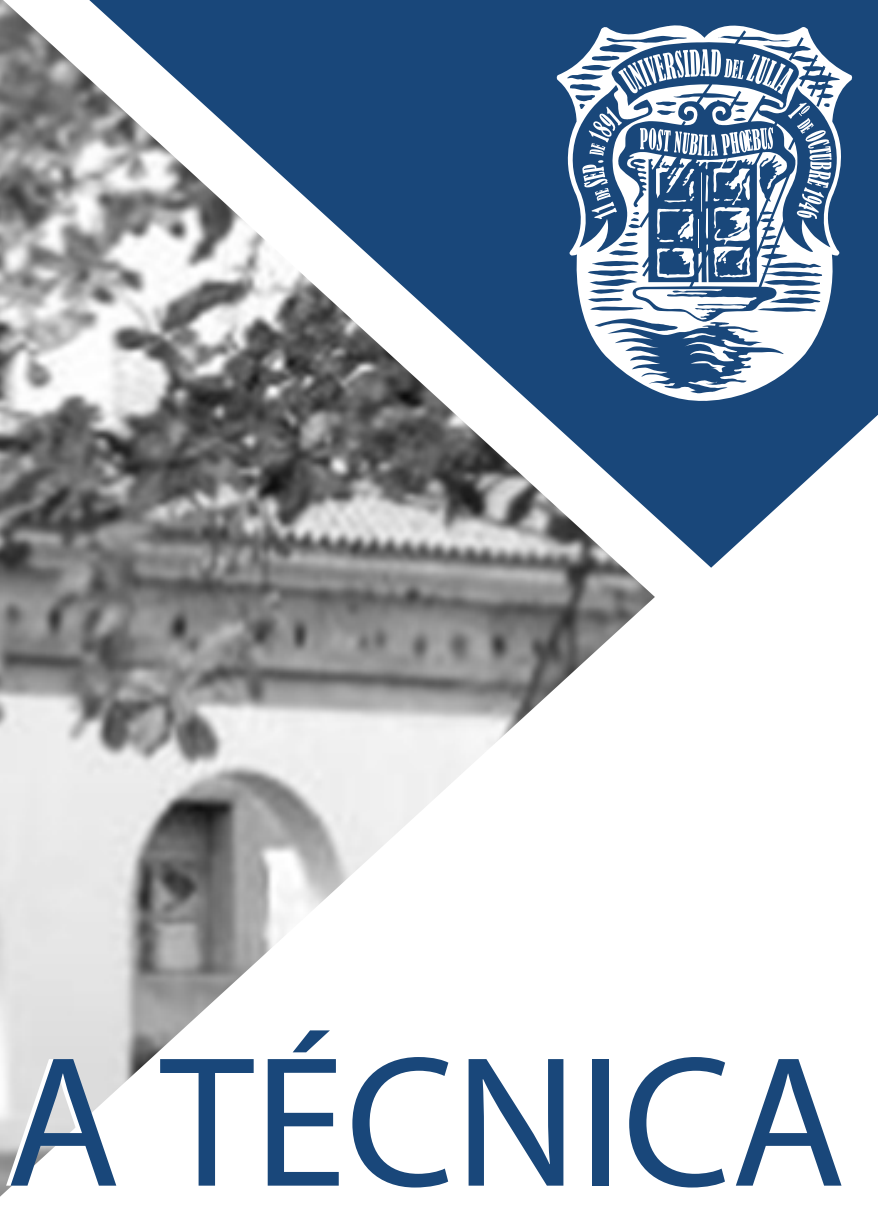
DE LA FACULTAD DE INGENIERÍA

Una Revista Internacional Arbitrada que está indizada en las publicaciones de referencia y comentarios:

- SCOPUS

- SCIELO

- LATINDEX

- DOAJ

- MIAR

- REDIB

- AEROSPACE DATABASE

- CIVIL ENGINEERING ABTRACTS

- METADEX

- COMMUNICATION ABSTRACTS

- ZENTRALBLATT MATH, ZBMATH

- ACTUALIDAD IBEROAMERICANA

- BIBLAT

- PERIODICA

- REVENCYT

UNIVERSIDAD DEL ZULIA 


\title{
Diseño, Fabricación y Caracterización de Celdas Solares p-CdTe/n-CdS con Películas Delgadas
}

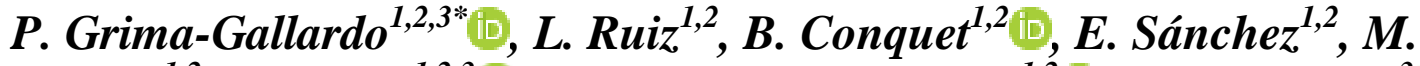

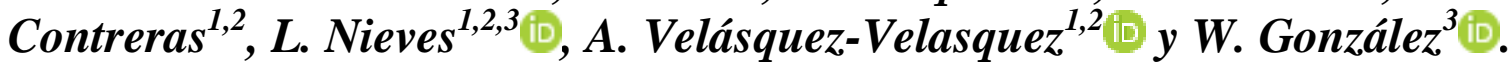 \\ ${ }^{1}$ Centro Nacional de Tecnologías Ópticas (CNTO). 5101 Mérida. Venezuela. \\ ${ }^{2}$ Centro de Investigaciones de Astronomía (CIDA). 5101 Mérida. Venezuela. \\ ${ }^{3}$ Centro de Estudios en Semiconductores (CES). Facultad de Ciencias. Departamento de \\ Física. Universidad de Los Andes. Mérida. Venezuela. \\ *Autor de correspondencia: peg1952@gmail.com \\ https://doi.org/10.22209/rt.v44n2a07 \\ Recepción: 12 de junio de 2020 | Aceptación: 30 de marzo de 2021 | Publicación: 30 de abril 2021
}

\section{Resumen}

Venezuela, debido a su ubicación geográfica, riqueza en fuentes y recursos naturales, tiene un valioso potencial para el uso de energías renovables, especialmente la solar. Gran parte de su territorio se caracteriza por una insolación media por encima de $3 \mathrm{Kwh} / \mathrm{m}^{2}$, considerada apta para la generación fotovoltaica. El CdTe es un conocido semiconductor II-VI, que se ha consolidado como líder en la industria fotovoltaica, posee propiedades óptimas para las celdas solares. Esta investigación se enfocó en diseñar, fabricar y caracterizar celdas solares pCdTe/n-CdS. El diseño se realizó buscando el modelo más simple y de menor costo. La fabricación se llevó a cabo en un equipo Ortus-700, usando la técnica de evaporación térmica. Para la caracterización de las celdas, se aplicaron las técnicas de difracción de rayos-X, absorción óptica y análisis de característica corriente-voltaje. Evidenciándose una orientación preferencial típica de una estructura cúbica de la Zinc Blenda, valores de brecha de energía directa entre 2,15 a 2,85 eV y un rendimiento similar entre las celdas solares preparadas. Se logró la fabricación y caracterización de nueve celdas p-CdTe/n-CdS, mediante la formación de una unión p-n; con eficiencias de $\sim 3 \%$.

Palabras clave: absorción óptica; celdas solares tipo p-CdTe/n-CdS; difracción de rayos X; eficiencia; películas delgadas.

\section{Design, Fabrication and Characterization of Thin-Film p-CdTe/n-CdS Solar Cells}

\begin{abstract}
Venezuela, due to its geographical location, richness in sources and natural resources, has a valuable potential for the use of renewable energies, especially solar. A large part of its territory is characterized by an average insolation above $3 \mathrm{Kwh} / \mathrm{m}^{2}$, considered suitable for photovoltaic generation. CdTe is a well-known II-VI semiconductor, which has established itself as a leader in the photovoltaic industry, has optimal properties for solar cells. This research focused on designing, manufacturing and characterizing $\mathrm{p}-\mathrm{CdTe} / \mathrm{n}-\mathrm{CdS}$ solar cells. The design was carried out looking for the simplest and lowest cost model. The manufacturing was carried out in an Ortus-700 equipment, using the thermal evaporation technique. For the characterization of the cells, the techniques of X-ray diffraction, optical absorption and current-voltage characteristic analysis were applied. Evidence of a typical preferential orientation of a cubic structure of Zinc Blenda, direct energy gap values between 2.15 to $2.85 \mathrm{eV}$ and similar performance among prepared solar cells. The manufacture and characterization of nine $\mathrm{p}-\mathrm{CdTe} / \mathrm{n}-\mathrm{CdS}$ cells was achieved, through the formation of a p-n junction; with efficiencies of $\sim 3 \%$.
\end{abstract}

Keywords: efficiency; optical absorption; p-CdTe/n-CdS solar cells; thin films; X-ray diffraction. 


\section{Introducción}

Venezuela, debido a su ubicación geográfica y riqueza en fuentes y recursos naturales, tiene un valioso potencial para el uso de energías renovables, especialmente la solar. Como se puede observar en la Figura 1, un gran porcentaje del territorio nacional se caracteriza por una insolación media del orden de $4-5 \mathrm{kWh} / \mathrm{m}^{2}$ por día (Solargis, 2017). Teniendo en cuenta que valores superiores a $3 \mathrm{kWh} / \mathrm{m}^{2}$ (Posso et al., 2014), se consideran aptos para la generación fotovoltaica se puede concluir que Venezuela es un país donde la explotación de la energía solar es muy conveniente. Además, se ha estimado que la insolación diaria en el territorio equivale a 4,56 millones de barriles de petróleo, cantidad que supera la producción diaria de petróleo de Venezuela en cualquier momento de su historia (Posso et al., 2014).

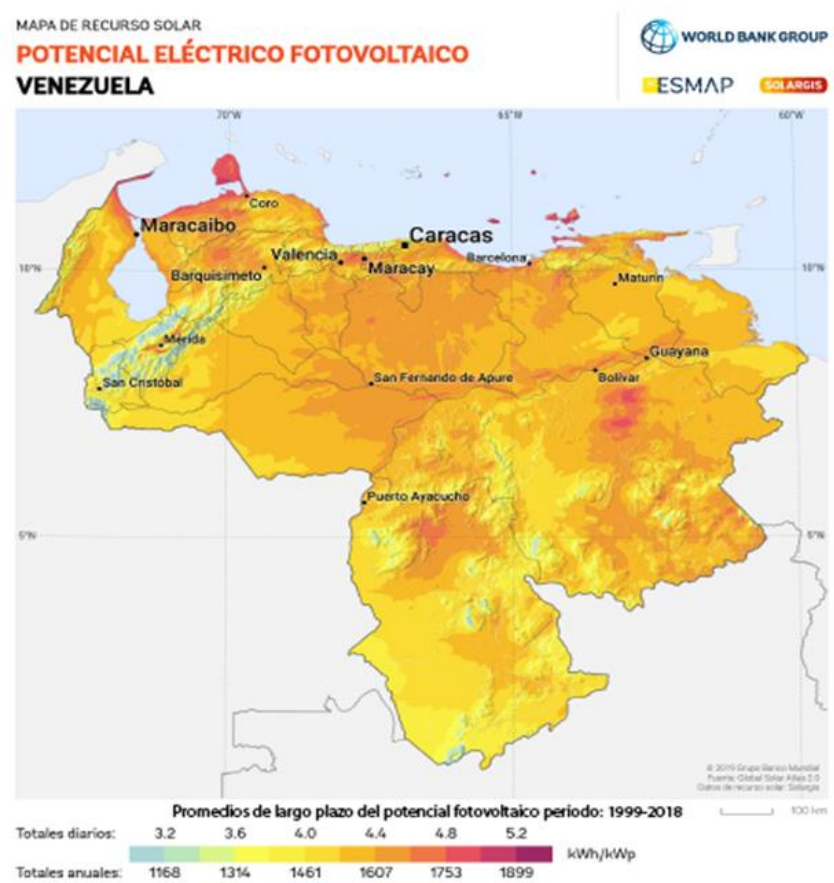

Figura 1. Mapa de recursos solares de Venezuela (modificado de Solargis, 2017).

Existen muchos tipos de celdas solares (Bagher et al., 2019), sin embargo, desde el punto de vista de su producción industrial (Figura 2), se pueden clasificar entre grandes grupos: 1) silicio poli-cristalino, 2) silicio mono-cristalino, y 3) películas delgadas (Phillips y Warmuth, 2016). Como se puede observar en la Figura 2, la tecnología de películas delgadas ha ido creciendo en los últimos años, particularmente las celdas basadas en CdTe (Figura 3).

Aproximadamente $57 *$ GWp de producción de módulos fotovoltaicos en 2015

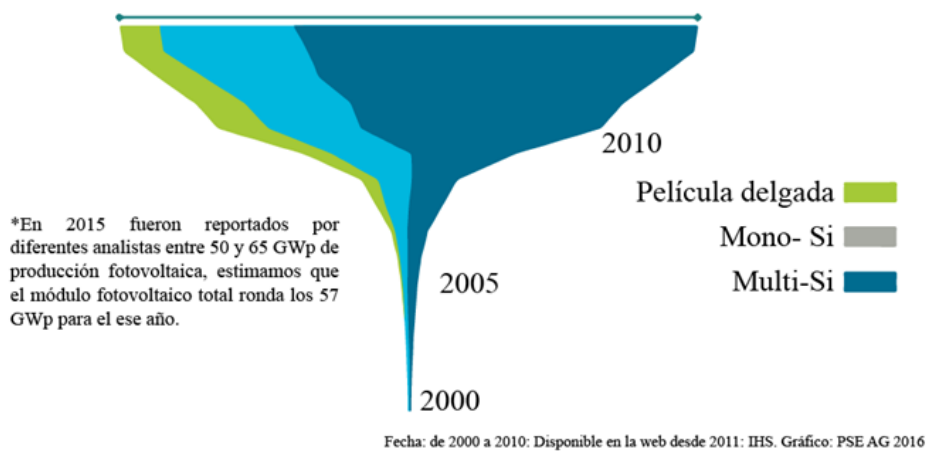

Figura 2. Producción mundial fotovoltaica por tecnología (en GWp) (modificado de Phillips y Warmuth, 2016). 


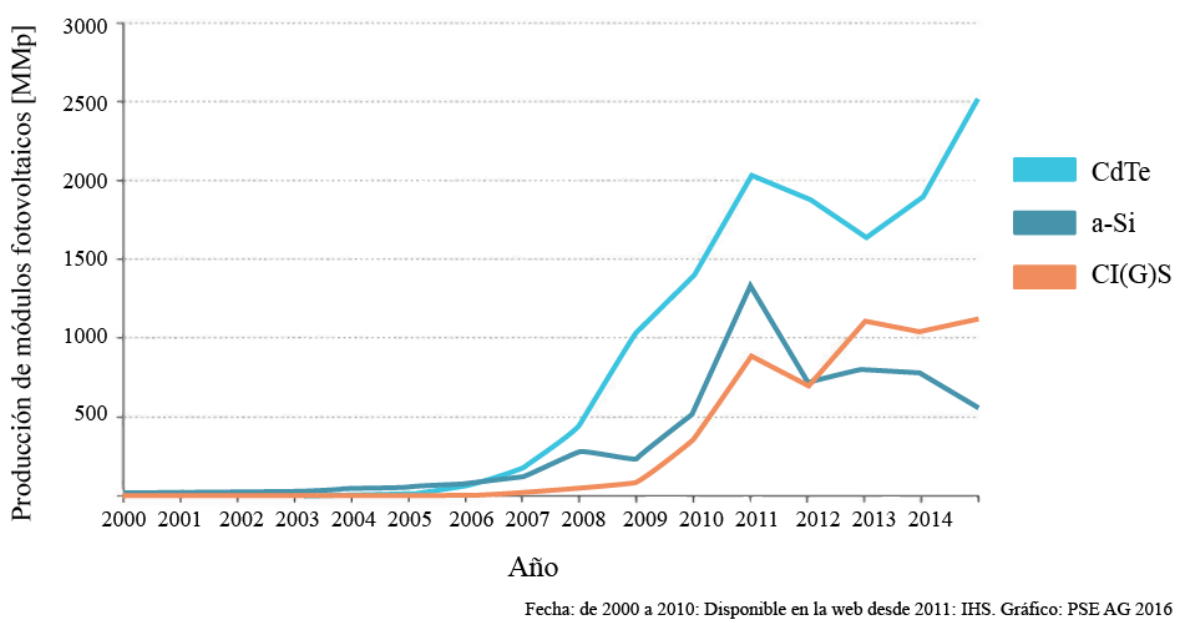

Figura 3. Tecnologías de película fina: producción anual mundial de módulos fotovoltaicos (modificado de Phillips y Warmuth, 2016).

El CdTe (teluro de cadmio) es un conocido semiconductor II-VI (Strauss, 1977), que tiene propiedades óptimas para las celdas solares. Las películas delgadas de CdTe tienen un alto coeficiente de absorción óptica que generalmente supera los $10^{4} \mathrm{~cm}^{-1}$, absorbiendo aproximadamente el $92 \%$ de la luz visible en un espesor de solo $1 \mu \mathrm{m}$ (Strauss, 1977; Rangel y Sobral, 2017); esta eficiencia está muy por encima del silicio cristalino, que necesita alrededor de $200 \mu \mathrm{m}$ para alcanzar el mismo valor de absorción (Burgelmn, 2006). Además, tiene un gap de energía óptica directa de 1,606 eV a temperatura de helio líquido y de $1,529 \mathrm{eV}$ a temperatura ambiente, óptima para acoplarse con el espectro de radiación solar (Strauss, 1977; Rangel y Sobral, 2017). También, sus elementos constituyentes, Cd y Te, todavía son relativamente abundantes en la corteza terrestre; esto significa que el costo comercial del polvo de CdTe en el mercado internacional es del orden de $\$$ 245/kg (pureza 4 N) y $\$ 280 / \mathrm{kg}$ (5 N) (Alibaba, 2017). De hecho, el CdTe se ha consolidado como líder en la industria fotovoltaica de película fina, logrando eficiencias de hasta el $22 \%$ gracias a la optimización de la densidad de corriente de cortocircuito $\left(\mathrm{J}_{\mathrm{sc}}\right)$, que ha pasado en los últimos años de 26,1 a $31,69 \mathrm{~mA} / \mathrm{cm}^{2}$ (Green et al., 2017).

El CdS también es un semiconductor bien conocido de la familia II-VI (Bube, 2001), naturalmente de tipo $\mathrm{n}$, con un coeficiente de absorción óptica, $\alpha$, de $\sim 1,1 \times 10^{5} \mathrm{~cm}^{-1}$, transparente, con una brecha de energía óptica directa de 2,41 eV (Oliva et al., 2001; Das y Pandey, 2011). Por sus características ópticas y bajo costo (\$ 50-100/kg) (Alibaba, 2016) es el material de ventana más utilizado para celdas solares con CdTe.

El objetivo de esta investigación consistió en diseñar, fabricar y caracterizar celdas solares p-CdTe/nCdS; usando un diseño, versátil y sencillo, para que pueda ser replicado con facilidad, y con el menor costo posible, librando así la brecha de la imposibilidad del fácil acceso a la mayoría de los materiales requeridos para la fabricación de estos dispositivos fotovoltaicos.

\section{Materiales y Métodos}

\section{Diseño de las celdas solares}

El diseño de la celda experimental usado para la fabricación de la celda solar, en el presente trabajo, se muestra en la Figura 4.

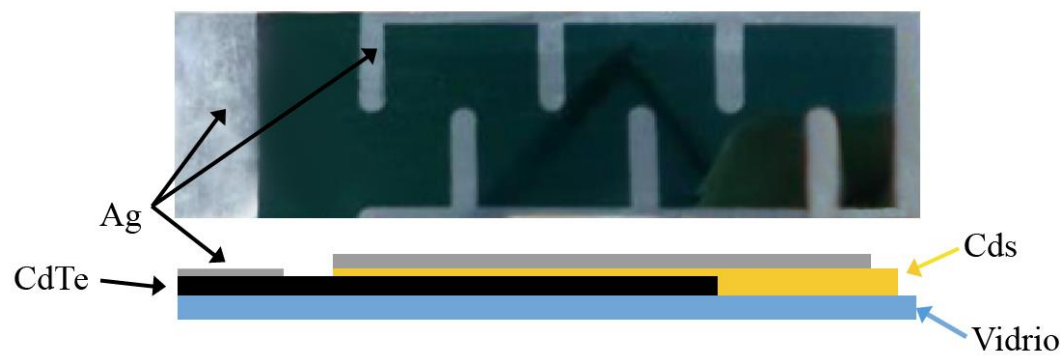

Figura 4. Detalles del diseño de la celda de p-CdTe/n-CdS. En la parte inferior, el arreglo experimental utilizado en este trabajo. En la parte superior, fotografía de la celda ya realizada. 


\section{Fabricación de las celdas solares}

El equipo utilizado para la fabricación de las nueves celdas de p-CdTe/n-CdS, fue un Ortus-700 (del Centro Nacional de Tecnologías Ópticas adscrita al Centro de Investigaciones de Astronomía Mérida, Venezuela), que permite obtener películas delgadas mediante cuatro técnicas diferentes: evaporación térmica, pistola de electrones, pistola de iones y radiofrecuencia. El sustrato consistió en portaobjetos de vidrio para microscopios, con dimensiones de 25,4×76,2 x1 mm. Antes de la deposición, la cámara se sometió a niveles de vacío del orden de $\sim 10^{-4}$ Torr; una vez alcanzados, los sustratos se sometieron a una limpieza con haz de iones ABS (Assisting Beam Source) durante 2 min, para eliminar las impurezas y lograr una superficie nanométrica rugosa que facilita la adherencia de materiales.

Para el proceso de deposición de CdTe, se utilizó polvo policristalino con una pureza nominal de $5 \mathrm{~N}$ $(99,999 \%)$, el cual se dispuso en forma de gránulos con un valor medio aproximado de 3 a 5 mm de diámetro cada pieza, dentro de un recipiente fabricado con molibdeno (Mo), que a su vez estaba conectado al par de electrodos del dispositivo de evaporación térmica dentro del Ortus-700.

Las películas se obtuvieron calentando gradualmente el CdTe dentro del crisol hasta que se evaporó. Las moléculas de vapor, saliendo de la fuente, se depositaron sobre la superficie del sustrato. El equipo cuenta con un soporte para los sustratos en forma de casquete esférico, lo que hace que la distancia entre la fuente calefactora y los sustratos varíe entre 45 y $53 \mathrm{~cm}$, según su ubicación en dicho soporte (Figura 5). Para obtener películas homogéneas, se utilizó la rotación mecánica de dicho soporte, el cual se configuró para girar a 20 rpm, y se ajustó la potencia de calentamiento hasta alcanzar tasas de deposición de 2-6 ̊̊/s.

La nomenclatura 1, m y c de la Figura 5 es arbitraria, permitiendo la evaluación posterior de la diferencia de espesor de las películas en función de la posición, ya que la concavidad del soporte hace que las muestras ubicadas en la parte más excéntrica estén más cerca de la fuente.

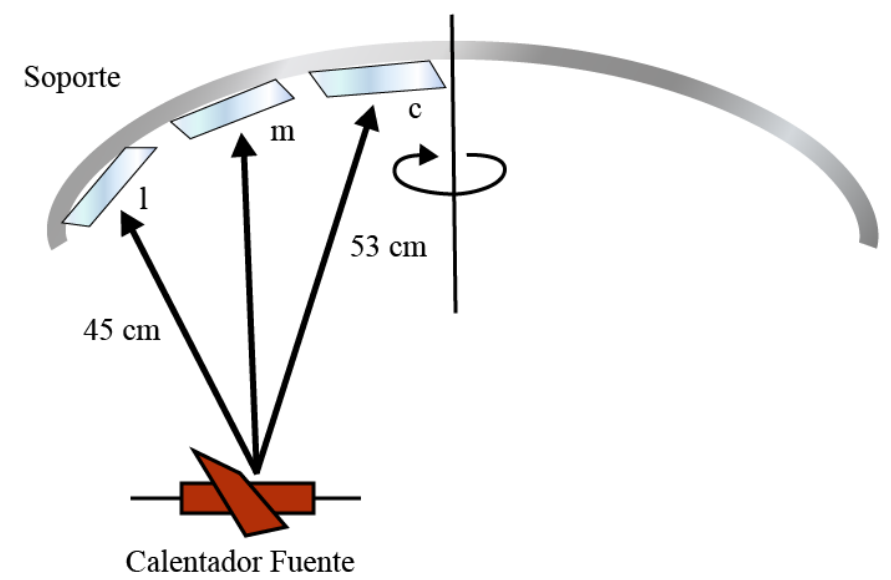

Figura 5. Vista lateral del soporte de los sustratos en el Ortus-700. La nomenclatura 1, m y c es arbitraria y permite evaluar posteriormente la diferencia de espesor de las películas en función de dicha posición (modificado de Izovac, 2014).

Para el proceso de deposición de CdS, se utilizó polvo policristalino con una pureza nominal de $5 \mathrm{~N}$ $(99,999 \%)$, que se sinterizó en tabletas de $25 \mathrm{~mm}$ de diámetro y un espesor de 0,5-0,8 $\mathrm{mm}$. De manera similar al compuesto CdTe, se colocaron fragmentos de dichos comprimidos dentro de un recipiente de Mo, conectado a los electrodos del dispositivo de evaporación térmica dentro del sistema de recubrimiento. En este caso, el sustrato consistió en las muestras de película fina de CdTe, obtenidas previamente. Antes de la deposición, la cámara también se sometió a un vacío del orden de $\sim 10^{-4}$ Torr, limpiando las muestras de CdTe por haz de iones ABS durante 2 min.

Las películas delgadas de CdS también se obtuvieron mediante la técnica de calentamiento resistivo, donde el sustrato durante la deposición alcanzó temperaturas similares a las de CdTe. Se utilizó la rotación mecánica a $20 \mathrm{rpm}$ y se ajustó la potencia de calentamiento hasta alcanzar una tasa de deposición de $3 \AA$ Ås y un espesor de $\sim 500 \mathrm{~nm}$, medido in situ por transmisión óptica, que corresponde a $\mathrm{T}=50 \%$ para radiación incidente con $\lambda=550 \mathrm{~nm}$. Una vez dispuestas las capas de CdTe y CdS sobre el sustrato de vidrio, se fijaron sobre ellas los contactos tipo dedos de plata (Ag), que se obtuvieron mediante la técnica de deposición por haz de electrones, alcanzando un espesor de unos $350 \mathrm{~nm}$. 


\section{Caracterización de las celdas solares}

La primera caracterización realizada fue la medición de las películas por difracción de rayos X (XRD), mediante la técnica de polvo, utilizando un equipo Siemens Brukers-AXS D5005. La segunda caracterización realizada fue la medida de la transmisión óptica, en función de la longitud de onda incidente para las películas obtenidas, en equipos Cary-UV-Vis-NIR-5000 a incidencia normal. La tercera caracterización que correspondió a la medición de la eficiencia de las celdas, utilizando su característica densidad de corriente-tensión (J-V), se presenta en la Figura 6. En el lado izquierdo se muestra el circuito de medición utilizado. Los valores de $\mathrm{R}_{\mathrm{L}}$ adecuados pueden estimarse mediante el siguiente procedimiento. Primero, se midieron $\mathrm{V}_{\mathrm{oc}}$ y $\mathrm{J}_{\mathrm{sc}}$, y se calculó la relación de $V_{o c}, J_{s c} y R_{L} *$. El valor de $R_{L} *$ está cerca de $R_{L}$ en el punto de máxima potencia. Se eligieron valores de resistencias menores o mayores que $\mathrm{R}_{\mathrm{L}}$ *, en aproximadamente un orden de magnitud o más para obtener puntos en las características JV hacia $J_{s c}$ o hacia $V_{o c}$, respectivamente, donde se deben elegir más valores de $R_{L}$ alrededor del punto de máxima potencia para aumentar la precisión de la medición del factor de llenado (FF), definido a continuación (Dittrich, 2018). En el lado derecho de la Figura 6, también se muestra la estación casera que se utilizó para manipular los contactos eléctricos a la celda solar. Se usaron dos brazos de bronce para sostener el cable necesario para cada material semiconductor. Para una respuesta adecuada se utilizaron contactos óhmicos. El contacto CdTe consistió de alambre de cobre de 0,5 mm que se soldó a la superficie de la película delgada con pintura plateada, mientras que para el CdS se utilizó un alambre de tungsteno de 0,2 $\mathrm{mm}$ de diámetro. El comportamiento óhmico de los electrodos se verificó midiendo la característica densidad de corriente-voltaje.
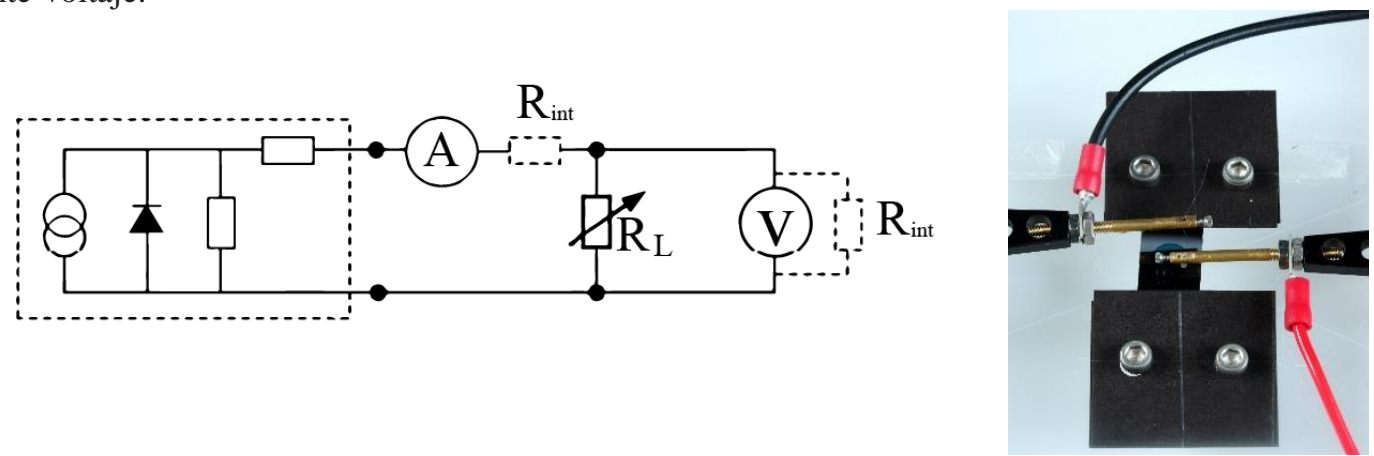

Figura 6. Disposición del circuito eléctrico usado para la medición de las características de corriente y voltaje $(\mathrm{J}-\mathrm{V})$, utilizando un potenciómetro y dos multímetros. $\mathrm{R}_{\mathrm{int}}$ : resistencia interna, $\mathrm{R}_{\mathrm{L}}$ : resistencia variable, A: amperímetro, V: voltímetro.

Las lecturas de voltaje y corriente se tomaron usando dos instrumentos de medición Keithley 2400. Las curvas J-V se obtuvieron punto a punto bajo iluminación. La luz incidente fue proporcionada por una bombilla de luz incandescente de tungsteno (eficacia luminosa de 12,5-17,5 lúmenes/vatio), que se colocó a una distancia de alrededor de $1 \mathrm{~m}$ sobre la muestra de la célula solar, para evitar un calentamiento indeseable del dispositivo; la luminosidad se midió con un luxómetro comercial PCL-VDL $16 \mathrm{I}$.

La eficiencia $(\eta)$ de una celda solar se determina como la fracción de energía incidente que se convierte en electricidad, y se define como (Checa et al., 2015):

$$
\eta=\frac{P_{\text {máx }}}{P_{\text {in }}}=\frac{V_{o c} J_{s c} F F}{P_{\text {in }}}
$$

Donde $\mathrm{P}_{\text {in }}$ es la potencia incidente, $\mathrm{V}_{\mathrm{oc}}$ es el voltaje de circuito abierto; $\mathrm{J}_{\mathrm{sc}}$ es la densidad de corriente de cortocircuito y FF es el factor de llenado, dado por:

$$
F F=\frac{V_{\text {máx }} J_{m a ́ x}}{V_{o c} J_{S c}}
$$

Donde $\mathrm{V}_{\text {máx }} \mathrm{y} \mathrm{J}_{\text {máx }}$ son las coordenadas del punto $\mathrm{P}_{\text {máx }}$.

La potencia incidente $\mathrm{P}_{\text {in }}\left[\mathrm{W} / \mathrm{cm}^{2}\right]$ es igual al flujo luminoso $\Phi_{\mathrm{V}}$ [lúmenes $\left./ \mathrm{cm}^{2}\right]$, dividido por la eficacia luminosa $\mathrm{L}_{\mathrm{ef}}[$ lúmenes/W]:

$$
P_{i n}=\Phi_{V} / L_{e f}
$$

En este caso, la potencia incidente medida sobre la celda solar $P_{i n}=0,1 \mathrm{~W} / \mathrm{cm}^{2}$. 


\section{Resultados y Discusión}

En la Figura 7 se muestra el patrón de difracción obtenido a partir de la película de CdTe, El patrón de difracción muestra una orientación preferencial del plano 111, típica de una estructura cristalina cúbica, similar a la Zinc Blenda (Datta et al., 2011), estructura cristalina del CdTe. En la Figura 8 se muestra el patrón de difracción de CdS. La comparación del patrón experimental (rojo) con los calculados (azul y verde,) muestra inequívocamente que la estructura cristalina corresponde a la conocida fase hexagonal Wurtzite de CdS. Cabe señalar que los patrones corresponden a las películas a medida que se evaporaron, sin ningún tratamiento térmico posterior, debido a la buena cristalinidad durante su crecimiento.

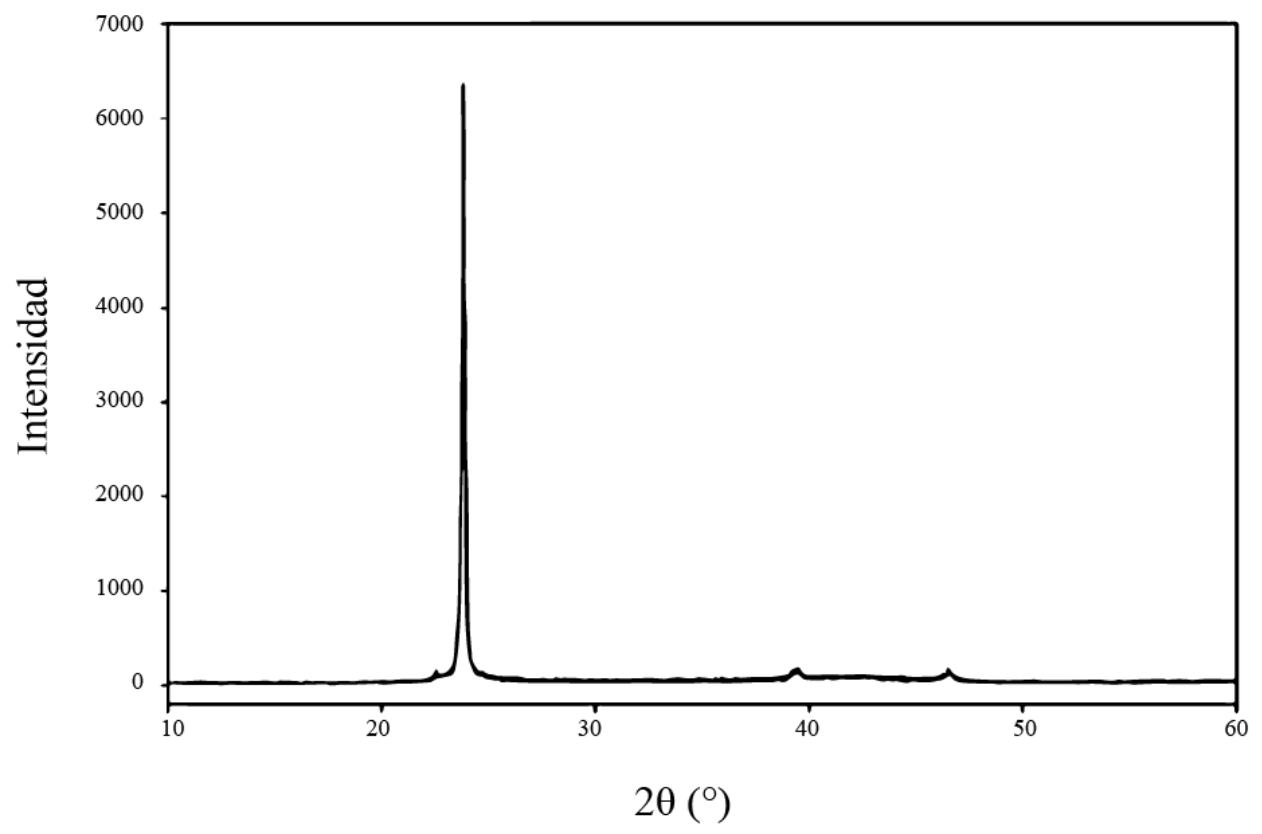

Figura 7. Patrón de difracción de rayos x, de la película delgada de CdTe fabricada en este estudio. $\left({ }^{\circ}\right.$ ): grados.

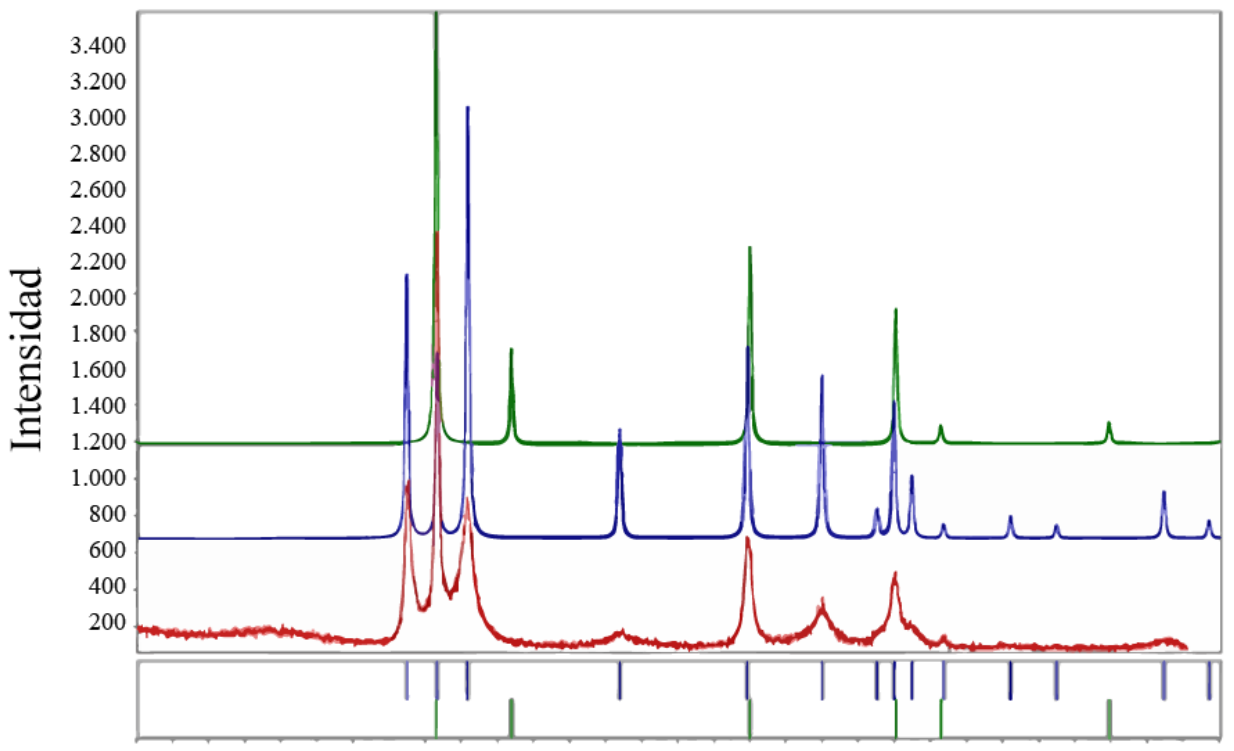

10121416182022242628303234363840424446485052545658606264666870

$2 \theta\left({ }^{\circ}\right)$

Figura 8. Patrón de difracción de película delgada de CdS (línea roja). A modo de comparación, también se muestran los patrones de difracción calculados de CdS hexagonal (línea azul: ICDD 01-074-9663) y CdS cúbico (línea verde: ICDD 04-006-3897). $\left(^{\circ}\right)$ : grados.

En la Figura 9 se muestran las medidas de las nueve películas de CdTe; cada color agrupa tres medidas diferentes que prácticamente se superponen. Cada color (negro, gris y azul) representa las posiciones 1, 
m y c en la Figura 6 y, por lo tanto, tres espesores diferentes de las películas: 355, 220 y 90 nm, para las curvas de color negro, gris y azul, respectivamente.

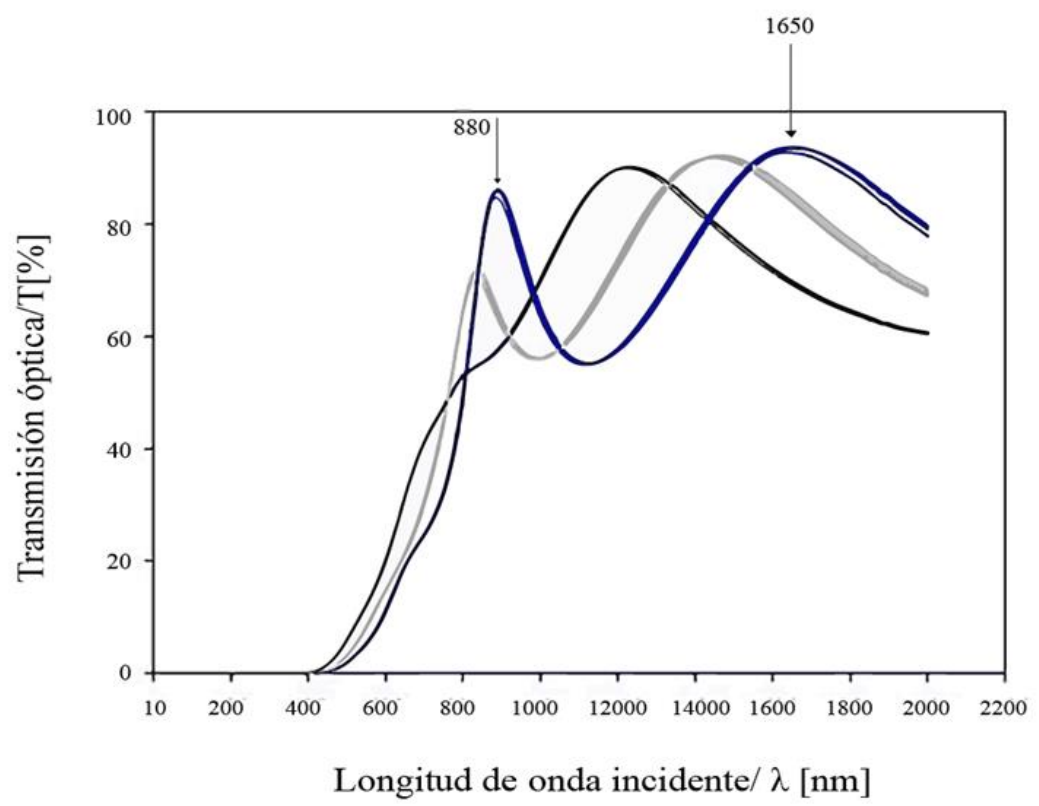

Figura 9. Medidas de transmisión óptica en función de la longitud de onda incidente para las nueve películas delgadas de CdTe. Los tres colores (negro, gris y azul) corresponden a las posiciones 1, m y c en la Figura 5, respectivamente. Observe que hay tres curvas superpuestas para cada posición. Los números de la curva muestran los valores de las dos bandas de absorción observadas.

Se conoce que el valor de la brecha de energía (Eg) depende del espesor de la película (Oliva et al., 2001; Goh et al., 2010; Khatri et al., 2010; Das y Pandey, 2011; Brus, L., 1984). En la Figura 10 se han graficado los valores de Eg vs $\lambda$ obtenidos de la Figura 9, con los valores de los espesores de las películas de CdTe. Se observa un comportamiento tipo exponencial $\mathrm{y}=\mathrm{Ax}^{-\mathrm{b}}$, con $\mathrm{A}=5,68 \mathrm{eV}$ y b=0,195, que coincide con lo observado por otros autores, por ejemplo para Ge (Goh et al., 2010), InSbBi (Khatri et al., 2010) y CdS (Oliva et al., 2001; Das y Pandey, 2011).

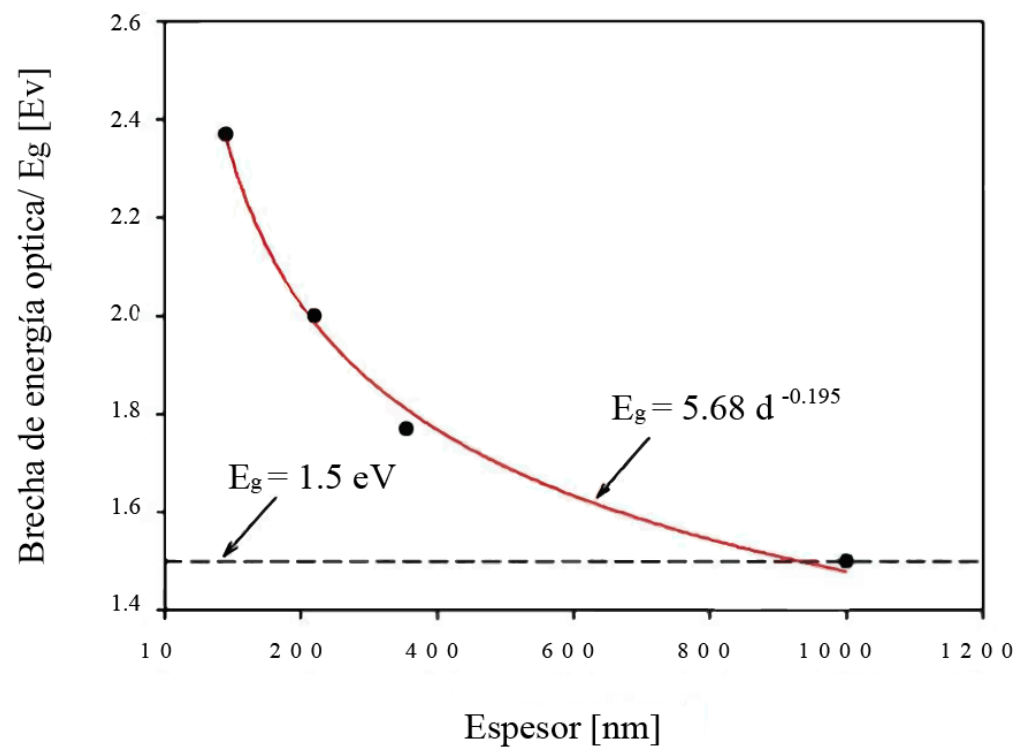

Figura 10. Brecha de energía (Eg) vs espesor de película para CdTe. Puntos negros: valores experimentales, excepto el punto a $1.000 \mathrm{~nm}$ que se ha tomado de la literatura (Hixum et al., 2009). La línea punteada indica el valor asintótico de $\mathrm{E}_{\mathrm{g}}$ para $\mathrm{CdTe}$ en bulto, mientras que la línea roja es un ajuste con una función del tipo $\mathrm{y}=\mathrm{Ax}^{-\mathrm{b}}$. 
En la Figura 11 se muestran los resultados de las nueve películas de CdS. En este caso, todas las curvas se superponen, por lo que no se observa ningún cambio de la brecha de energía. Este resultado indica que el espesor de las películas es el mismo: $500 \mathrm{~nm}$, medido in situ en el momento de la deposición. El valor de $\mathrm{E}_{\mathrm{g}}=$ $2,59 \mathrm{eV}$, se encuentra dentro del rango de los valores obtenidos por otros autores, también en películas delgadas: 2,15-2,25 eV (Demir y Gode, 2015); 2,85 eV (Imran et al., 2018) y 2,42 eV (Oliva et al., 2001).

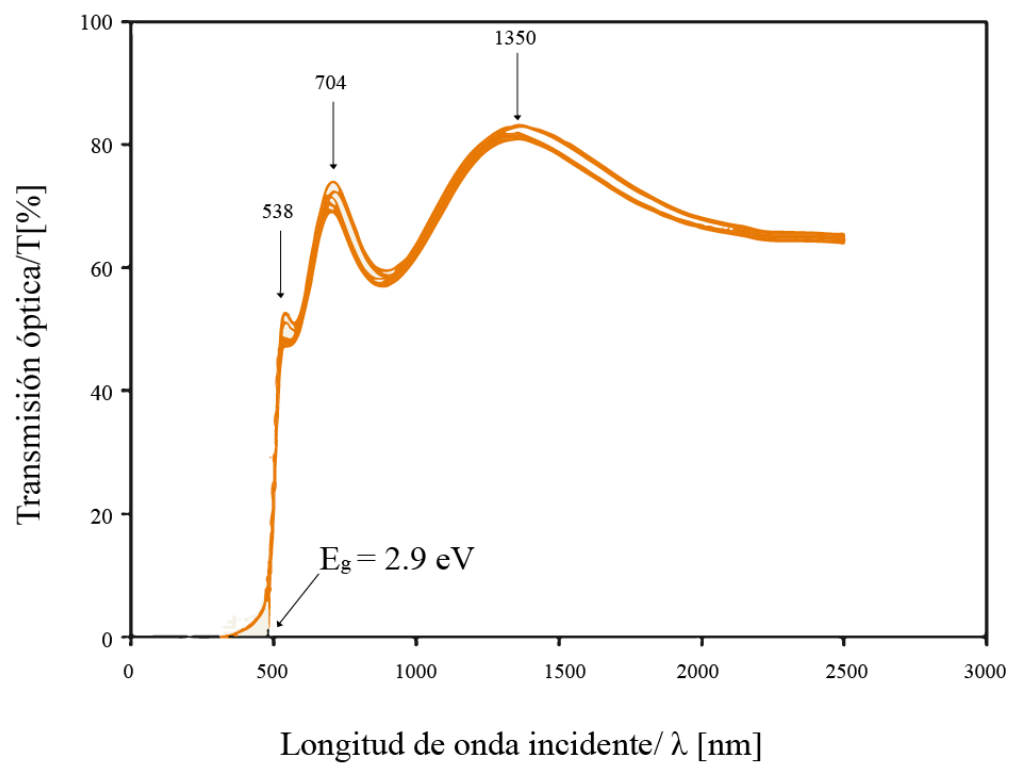

Figura 11. Medidas de transmisión óptica en función de la longitud de onda incidente para las nueve películas delgadas de CdS. Los números de la curva son los valores de las bandas de absorción observadas. También se muestra el valor de la brecha de energía directa $\left(\mathrm{E}_{\mathrm{g}}\right)$.

La caracterización corriente-tensión (J-V) para las nueve celdas, se expone en la Figura 12; la densidad de corriente $\mathrm{J}\left[\mathrm{mA} / \mathrm{cm}^{2}\right]$ y la salida de potencia $\mathrm{P}_{\text {out }}\left[\mathrm{mW} / \mathrm{cm}^{2}\right]$, se representan como una función del voltaje aplicado $\mathrm{V}[\mathrm{mV}]$ para una celda en particular (celda número 1). En la Tabla 1, se presentan los parámetros característicos de las nueve celdas.

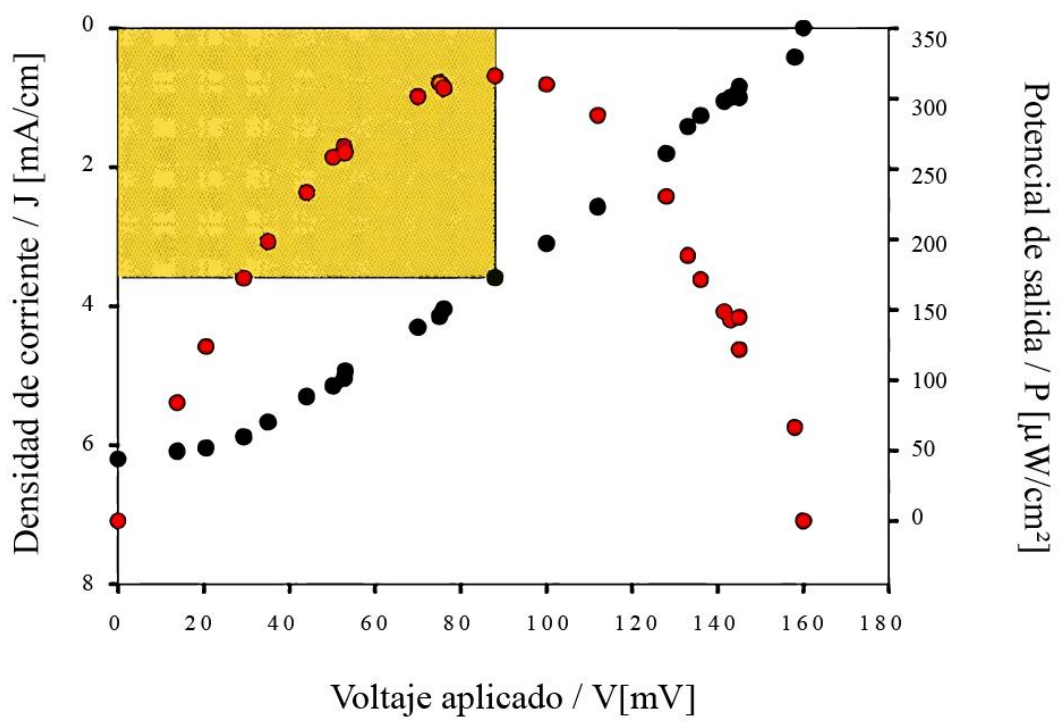

Figura 12. Característica de corriente-voltaje para la celda solar p-CdTe/n-CdS identificada con el número1 en la Tabla 1. Los puntos negros son los valores experimentales de J vs V. Los puntos rojos son la potencia de salida. El recuadro amarillo representa el factor de relleno (FF). 
Tabla1. Comportamiento fotovoltaico de las celdas solares p-CdTe/n-CdS fabricadas en el presente estudio.

\begin{tabular}{ccccccc}
\hline $\begin{array}{c}\text { Celda solar } \\
\text { número }\end{array}$ & $\begin{array}{c}\mathbf{V}_{\text {máx }} \\
{[\mathbf{m V}]}\end{array}$ & $\begin{array}{c}\mathbf{J}_{\text {máx }} \\
{\left[\mathbf{m A} / \mathbf{c m}^{2}\right]}\end{array}$ & $\begin{array}{c}\mathbf{V}_{\text {oc }} \\
{[\mathbf{m V}]}\end{array}$ & $\begin{array}{c}\mathbf{J}_{\mathbf{s c}} \\
{\left[\mathbf{m A} \mathbf{\mathbf { c m } ^ { 2 } ]}\right.}\end{array}$ & $\begin{array}{c}\mathbf{F F} \\
{[\%]}\end{array}$ & $\begin{array}{c}\eta \\
{[\%]}\end{array}$ \\
\hline 1 & 88 & 3,6 & 160 & 6,2 & 32 & 3,2 \\
2 & 95 & 3,5 & 191 & 5,0 & 35 & 3,3 \\
3 & 92 & 3,5 & 178 & 5,8 & 31 & 3,2 \\
4 & 93 & 3,4 & 175 & 5,9 & 36 & 3,2 \\
5 & 101 & 3,1 & 181 & 5,4 & 32 & 3,1 \\
6 & 99 & 3,1 & 178 & 5,6 & 31 & 3,1 \\
7 & 99 & 3,5 & 169 & 5,9 & 35 & 3,5 \\
8 & 97 & 3,6 & 165 & 6,1 & 35 & 3,5 \\
9 & 91 & 3,4 & 163 & 6,2 & 31 & 3,1 \\
\hline
\end{tabular}

Vmáx: voltaje máximo; Jmáx: corriente máxima; Voc: voltaje de circuito abierto; Jsc: corriente de circuitos cerrado, FF: factor de llenado; $\mathrm{y}$ : eficiencia de conversión.

Como se puede observar en la Tabla 1, el rendimiento de las celdas solares preparadas es muy similar, esto se debe a que todas fueron preparadas siguiendo la misma metodología y bajo las mismas condiciones experimentales.

\section{Conclusiones}

La fabricación y caracterización de las nueve celdas p-CdTe/n-CdS, mediante la formación de una unión p-n capaz de generar una diferencia de potencial al ser iluminada (efecto fotovoltaico). Para este primer prototipo se aplicó un diseño simple y con el menor costo posible; lográndose eficiencias de $3 \%$. La corriente de cortocircuito fue relativamente baja, probablemente debido a un valor alto de la resistencia en derivación $\left(\mathrm{R}_{\underline{\underline{s}}}\right)$.

\section{Agradecimiento}

Agradecemos al Laboratorio de Cristalografía de la Universidad de Los Andes, por las mediciones de difracción de rayos X; al Fonacit por el financiamiento del proyecto "Medición y optimización de la eficiencia de las celdas solares fotovoltaicas fabricadas en el Centro Nacional de Tecnologías Ópticas (CNTO)” (2018), y al Ministerio del Poder Popular para la Ciencia y Tecnología, por el financiamiento del proyecto POA 2019 "Fabricación y montaje de un módulo fotovoltaico".

\section{Referencias Bibliográficas}

Alibaba. Nanoparticles CdS powder price. <https://www.alibaba.com/product-detail/Nanoparticles-CdSpowder-price-CAS-1306_60521158667.html.> [Accessed April 14, 2020].

Alibaba. price cadmium telluride. <https://spanish.alibaba.com/product-detail/pure-cdte-price-4n-5n-6n-instock-with-good-price-cadmium-telluride-62416992602.html.> [Accessed April 7, 2020].

Bagher, A., Abadi, M. and Mohsen, M., 2019. Types of Solar Panels (2021) | GreenMatch. Greenmatch.co.uk.<https://www.greenmatch.co.uk/blog/2015/09/types-of-solar-panels> [Accessed Abril 4, 2020].

Brus, L., 1994. Electron-electron and electron hole interactions in small semiconductor crystallites: The size dependence of the lowest excited electronic state. J. Chem. Phys., 80(9).

Bube, R., 2001. Encyclopedia of materials. Amsterdam: Elsevier, pp.873-879.

Burgelman, M. (2006). Cadmium Telluride Thin Film Solar Cells: Characterization, Fabrication and Modeling. In: Thin Film Solar Cells. Fabrication, Characterization and Applications. Poortmans, J.and Arkhipov, V.1st ed. IMEC, Leuven, Belgium. John Wiley \& Sons, Ltd, 277-324. ISBN: 0-470-09126-6. 
Checa F., E., O. \&amp, E. and Rosero E, E., 2015. Study of the relationship between irradiance and power genarated by PV panel based on different silicon technologies in the city of pasto. Power Quality Aplications IEEE Workshop.1-6.

Das, R. and Pandey, S., 2011. Comparison of optical properties of b and nano crystalline thin films of CdS using different precursors. International Journal of Materials Science and Applications, 1(1), 35-40.

Demir, R. and Gode F., 2015. Structural, optical and electrical properties of nanocrystalline CdS thin films grown by chemical bath deposition method. Chalcogenide Letters, 12(2), pp.43-50.

Datta, S., Saha- Dasgupta T. and Saima, S., 2011. Relatibe Stability of Zincblende and Wurtzite Structure in $C d x(x=S, S e, T e)$ Serie-A NMTO Study. arXIV 1106.

Dittrich, T., 2018. Materials Concepts for Solar Cells. WorldScientific. Helmholtz Center Berlin for Materials and Energy, 2, 27.

Goh, E., Chen, T., Sun, C. and Liu, Y., 2010. Thickness effect on the band gap and optical properties of germanium thin films. Journal of Applied Physics, 107(2), p.024305.

Green, M., Hishikawa, Y., Dunlop, E., Levi, D., Hohl-Ebinger, J. y Ho-Baillie, A., 2017. Solar cell efficiency tables (version 51). Progress in Photovoltaics: Research and Applications, 26(1). 3-12. <https://onlinelibrary.wiley.com/doi/abs/10.1002/pip.2978> [Accessed Abril 19, 2020].

Hixum Ma, Z., Man Yu, K., Walukiewicz, W., Y. Yu, P. and S Mao, S., 2009. Stain relaxation of CdTe films growing on lattice-mismatched sustrate. Appl Phys A., 96(379-384.).

Imran, A., Jiang, J., Eric, D., Zahid, M.N., Yousaf, M., Ahmad, A., Hassan, S.A. (2018). Efficiency enhancement through flat intermediate band in Quantum dot solar cell. Results in Physics, 10, 241-247, ISSN 2211-3797, https://doi.org/10.1016/j.rinp.2018.05.037.

Izovac Ltd.Vacuum Tecnological Equipament., 2014. RTUS-700. Intructional Manual, IM-0000033000. 1 st ed.

Khatri, R., Vyas, S., Shah, D., Patel, P., Jani, M. and Pandya, G., 2010. Study of Thickness Dependence Optical Bandgap of InSbBi Thin Films. International Journal of Physics and Applications, 2(3), pp.95-99.

Oliva, A., Solís-Canto, O., Castro-Rodríguez, R. and Quintana, P., 2001. Formation Of The Band Gap Of Cds Thin films growth by different techniques. Modern Physics Letters B. 15.671-674.

Phillips, S. and Warmuth, W., 2016. “Photovoltaics report”. [ebook] Fraunhofer Institute for Solar Energy Systems, ISE with support of PSE <https://www.ise.fraunhofer.de/content/dam/ise/de/documents/publications/studies/Photovoltaics-Report.pdf> [Accessed Abril 19, 2020].

Posso, F., González, J., Guerra, F., Gómez, H. (2014). Estimación del potencial de energía solar en Venezuela utilizando sistemas de información geográfica. Revista Geográfica Venezolana, 55(1), 27-43.

Rangel-Cardenas, J. and Sobral, H., 2017. Optical absorption enhancement in CdTe thin films by microstructuration of the silicon substrate. Materials (Basel), 607, 1st ed.

Solargis. 2019. Solar resource maps of Venezuela. <https://solargis.com/es/maps-and-gisdata/download/venezuela> [Accessed Abril 10, 2020].

Strauss, A., 1977. The physical properties of cadmium telluride. 12th ed. Revue Physique Appliquée.167-184. 


\section{REVISTA TECNICA}

DE LA FACULTAD DE INGENIERIA

UNIVERSIDAD DEL ZULIA

Vol. 44. №2, Mayo - Agosto, 2021

Esta revista fue editada en formato digital y publicada en Abril de 2021, por el Fondo Editorial Serbiluz, Universidad del Zulia. Maracaibo-Venezuela

www.luz.edu.ve

www.serbi.luz.edu.ve

www.produccioncientificaluz.org 